\title{
Solitonic Model of the Electron, Proton and Neutron
}

\section{Sladkov $\mathbf{P}^{*}$}

Independent Researcher, Russia

\begin{abstract}
In paper, which is submitted, electron, proton and neutron are considered as spherical areas, inside which monochromatic electromagnetic wave of corresponding frequency spread along parallels, at that along each parallel exactly half of wave length for electron and proton and exactly one wave length for neutron is kept within, thus this is rotating soliton. This is caused by presence of spatial dispersion and anisotropy of strictly defined type inside the particles. Electric field has only radial component, and magnetic field - only meridional component. By solution of corresponding edge task, functions of distribution of electromagnetic field inside the particles and on their boundary surfaces were obtained. Integration of distribution functions of electromagnetic field through volume of the particles lead to system of algebraic equations, solution of which give all basic parameters of particles: charge, rest energy, mass, radius, magnetic moment and spin.
\end{abstract}

Keywords: Structure of elementary particles; Structure of matter; Theory of elementary particles; Electron; Proton; Neutron; Nuclei; Electromagnetic field; Atom; Microcosm; Elementary particles; Fundamental interactions; New theory; New physical theory

\section{Introduction}

In present article alternative (to Standard Model) hypothesis of structure of electron, proton and neutron is suggested. The others elementary particles (except photon and neutrino) are not stable and they are considered as unsteady soliton-similar formations. In series of experiments indirect confirmations of existence of quarks were obtained, for instance in experiments by scattering of electrons at nuclei, performed at Stanford linear accelerator by R. Hofshtadter, look for instance [1]. At that, experiments by elastic and deeply inelastic scattering gave quite different results: in first case take place pattern of scattering at lengthy object, in second case is pattern of scattering at "point" centers, that is interpreted as confirmations of existence of quarks. However what "point" formations appear only in deeply inelastic scattering don't may be an evidence of quarks existence, because to above-mentioned fact may be given and another explanations: in moment of birth of new particles, which take place in deeply inelastic scattering, structure of nucleon change, it sharply diminish in volume, but after appearance of new particles nucleon return to initial state. Or process of birth of new particles occur in "point" volume inside nucleon and these energy "point" centers disappear after completion of process particles birth. And fact that experiments by elastic scattering gave pattern of scattering at lengthy object prove inexistence of quarks in nucleus. In theory of Standard (quarkual) Model come into at least 20 parameters artificially introduced from outside, such as "colour" of particles, "aroma" etc., that is its fundamental demerit. Theoretical work, which is present here, has no demerits of Standard Model, it completely describe structure of elementary particles therefore it can help in discovery new ways of making energy, elaboration perfectly new devices for its production and to achieve progress in such fields as nuclear power engineering, nanotechnology, high-powerful lasers, clean energy and others.

\section{Rotating Monochromatic Electromagnetic Wave}

Let us write down Maxwell's equations in spherical coordinates supposing that:

1) There are no losses;

2) Only $\dot{E}_{r}, \dot{H}_{\theta}, \dot{j}_{\phi}, \dot{\rho}$ are not equal to zero.

$$
\begin{aligned}
& \frac{1}{r}\left(\frac{\partial}{\partial r}\left(r \dot{H}_{\theta}\right)\right)=\dot{j}_{\phi} ; \\
& \frac{1}{r} \frac{\partial \dot{E}_{r}}{\partial \theta}=i \omega \mu \dot{H}_{\phi}=0 ; \\
& \frac{1}{r} \frac{\partial \dot{E}_{r}}{\partial \theta}=i \omega \mu \dot{H}_{\phi}=0 ; \\
& \frac{1}{r \sin \theta}\left(-\frac{\partial \dot{H}_{\theta}}{\partial \phi}\right)=i \omega \varepsilon \dot{E}_{r} ; \\
& \frac{1}{r^{2}} \frac{\partial}{\partial r}\left(r^{2} \varepsilon \quad \dot{E}_{r}\right)=\dot{\rho} ; \\
& \frac{1}{r \sin \theta} \frac{\partial}{\partial \theta}\left(\sin \theta\left(\mu \dot{H}_{\theta}\right)\right)=0 .
\end{aligned}
$$

Here $r, \theta, \phi$ spherical coordinates of the observation point; $\dot{E}_{r}$ и $\dot{H}_{\theta}$ - components of the electromagnetic field, $\dot{j}_{\phi}$ - density of electric current, $\dot{\rho}$ - volume charge density; $\omega$-circular frequency of field alteration $i$ - imaginary unit $\varepsilon$-dielectric permittivity - magnetic permeability Figure 1.

Substituting the expression for $\dot{H}_{\theta}$ from (2) in (4), we obtain:

$$
\begin{aligned}
& \frac{\partial}{\partial}+\varepsilon \mu \omega^{2} r^{2} \sin ^{2} \theta E=0 \\
& \frac{1}{r^{2} \sin ^{2} \theta} \frac{\partial^{2} \dot{E}_{r}}{\partial \phi^{2}}+\omega^{2} \varepsilon \mu \dot{E}_{r}=0
\end{aligned}
$$

This is Helmholtz homogeneous equation. Let us designate.

$$
\sqrt{\varepsilon \mu} \omega r \sin \theta=k_{1}-
$$

Wave number - General solution of Helmholtz equation:

$$
\dot{E}_{r}=E_{0} e^{-i k_{1} \phi}+E_{0} e^{i k_{1} \phi} \text {. }
$$

*Corresponding author: Sladkov P, Independent Researcher, Russia, Tel: 086958945; E-mail: sladkovpaul@gmail.com

Received June 09, 2016; Accepted August 19, 2016; Published August 23, 2016

Citation: Sladkov P (2016) Solitonic Model of the Electron, Proton and Neutron. J Phys Math 7: 193. doi: 10.4172/2090-0902.1000193

Copyright: @ 2016 Sladkov P. This is an open-access article distributed under the terms of the Creative Commons Attribution License, which permits unrestricted use, distribution, and reproduction in any medium, provided the original author and source are credited. 


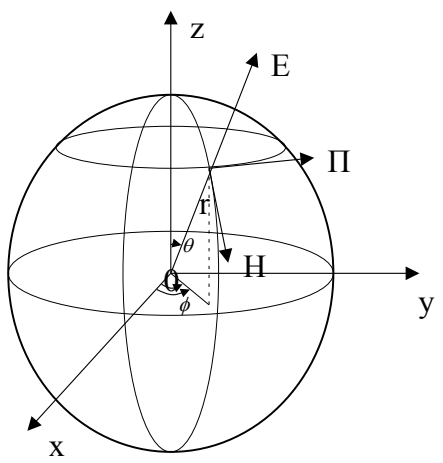

Figure 1: Representing the spherical coordinates of the observation point

This expression describes two waves, moving to meet one another by circular trajectories, along the parallels. Pointing's vector in each point is directed at tangent to the corresponding parallel $[2,3]$.

Let us consider a wave, moving in positive direction $\phi$.

$$
\dot{E}_{r}=E_{0} e^{-i k_{1} \phi} F(r, \theta) \text {; }
$$

Here

$$
k_{1} \phi=\sqrt{\varepsilon \mu}(\omega r \sin \theta) \phi-
$$

Wave phase;

$K_{1}$ - Dimensionless analog of the wave number. If to introduce a wave number of traditional dimension $\left(\frac{1}{m}\right)$;

$$
\beta=\omega \sqrt{\varepsilon \mu}=\frac{k_{1}}{r \sin \theta},
$$

The wave phase will be written down as

$k_{1} \phi=\sqrt{\varepsilon \mu} \omega(r \sin \theta) \phi=\beta l$,

Where

$$
l=(r \sin \theta) \phi-
$$

Arc length along the corresponding parallel. In the considered case the wave number is a function of coordinates and frequency. Thus, the wave, which is described, can exist only at availability of spatial and frequency dispersion [4-6]. Dispersion equations will be obtained below, apart from the already found expression $\left(7^{\prime \prime}\right)$.

From expression (2), taking into account ( $\left(7^{\prime \prime}\right)$ and (9), we have:

$$
\dot{H}_{\theta}=\frac{\sqrt{\varepsilon \mu} \omega r \sin \theta}{\mu \omega r \sin \theta} E_{0} e^{-i k_{1} \phi} F(r, \theta)=\frac{E_{0}}{z} e^{-i k_{1} \phi} F(r, \theta) .
$$

For actual amplitudes:

$$
\begin{aligned}
& E_{r}=E_{0} F(r, \theta) \quad \sin k_{1} \phi ; \\
& H_{\theta}=\frac{E_{0}}{z} F(r, \theta) \sin k_{1} \phi .
\end{aligned}
$$

Here

$$
z=\sqrt{\frac{\mu}{\varepsilon}}-
$$

Means characteristic impedance.

The last expressions describe an electromagnetic wave, rotating around axis $\mathrm{Z}$ in positive direction $\phi$. Conditions of self-consistency:

1) $z=$ constant
2) Along each parallel on the circle length, the integer number of half-waves must be kept within.

$2 \pi r \sin \theta=n \frac{\lambda}{2}$

Here $\lambda=\frac{v}{f}-$

Wave length, $\mathrm{v}$ - phase velocity of wave, $\mathrm{f}$ - frequency, $\mathrm{n}=1,2,3 \ldots$

Let us consider the case when $\mathrm{n}=1$,

$2 \pi r \sin \theta=\frac{\lambda}{2}$

$v=2 \omega r \sin \theta$

Along each parallel, exactly half of wave length is kept within.

Phase velocity of wave is the function of frequency and distance up to the axis of rotation.

$v=\frac{1}{\sqrt{\varepsilon \mu}}=2 \omega r \sin \theta ;$

$\varepsilon \mu=\frac{1}{4 \omega^{2} r^{2} \sin ^{2} \theta} ;$

$z=\sqrt{\frac{\mu}{\varepsilon}}$

$\mu=\varepsilon z^{2}$;

We are substituting in $\left(11^{\prime \prime}\right)$ :

$$
\begin{aligned}
& z=\frac{1}{2 \omega r \varepsilon \sin \theta} ; \\
& \varepsilon=\frac{1}{2 \omega r z \sin \theta} .
\end{aligned}
$$

From $\left(11^{\prime \prime}\right) \varepsilon=\frac{\mu}{z^{2}}$;

We are substituting in $\left(12^{\prime}\right)$.

$$
\begin{aligned}
& \mu=\frac{z}{2 \omega r \sin \theta} ; \\
& z=2 \mu \omega r \sin \theta .
\end{aligned}
$$

Taking into account (8) and (11")

$k_{1}=\frac{\omega r \sin \theta}{2 \omega r \sin \theta}=\frac{1}{2}$.

Then

$$
\begin{aligned}
& E_{r}=E_{0} F(r, \theta) \sin \frac{\phi}{2} ; \\
& H_{\theta}=\frac{E_{0}}{z} F(r, \theta) \sin \frac{\phi}{2} .
\end{aligned}
$$

Function $\sin \frac{\phi}{2}$ is on valued in angles interval $0 \leq \phi \leq 2 \pi$.

This situation can be interpreted as rotation of spherical coordinate system around axis $\mathrm{z}$ in positive direction $\phi$ with angular velocity $\frac{d \phi}{d t}$.
Let us find it from the condition:

$$
\omega t-\frac{\phi}{2}=\text { const }
$$

Having differentiated this expression on $\mathrm{t}$, we receive,

$$
\frac{d \phi}{d t}=2 \omega
$$

At the same time the electromagnetic field, about spherical 
coordinate system, is determined by expressions (13) and (13'). Further from (3): as $H_{\phi}=0$

$$
\begin{aligned}
& \dot{E}_{r}(\theta)=\text { const } ; \\
& E_{\mathrm{r}}(\theta)=\text { const }
\end{aligned}
$$

From equation (6)

$$
\frac{\partial}{\partial \theta}\left(\frac{\sin \theta\left(z H_{\theta}\right)}{2 \omega r \sin \theta}\right)=0
$$

Follows

$$
\begin{aligned}
& \dot{H}_{\theta}(\theta)=\text { const } ; \\
& H_{\theta}(\theta)=\text { const }
\end{aligned}
$$

To receive field dependence from $r: E_{r}(r) ; H_{\theta}(r)$, let us find solution of three-dimensional Helmholtz equation in spherical coordinates.

$$
\frac{1}{r^{2}} \frac{\partial}{\partial r}\left(r^{2} \frac{\partial E_{r}}{\partial r}\right)+\frac{1}{r^{2} \sin \theta} \frac{\partial}{\partial \theta}\left(\sin \theta \frac{\partial E_{r}}{\partial \theta}\right)+\frac{1}{r^{2} \sin ^{2} \theta} \frac{\partial^{2} E_{r}}{\partial \phi^{2}}+k^{2} E_{r}=0 .
$$

$E_{r}$ does not depend from $\theta$, look (14), therefore three-dimensional Helmholtz equation transfers into two-dimensional one.

$$
\frac{1}{r^{2}} \frac{\partial}{\partial r}\left(r^{2} \frac{\partial E_{r}}{\partial r}\right)+\frac{1}{r^{2} \sin ^{2} \theta} \frac{\partial^{2} E_{r}}{\partial \phi^{2}}+k^{2} E_{r}=0 \text {. }
$$

Let us suppose that

$$
k^{2}=k_{2}^{2}+k_{3}^{2},
$$

now

$\frac{1}{r^{2}} \frac{\partial}{\partial r}\left(r^{2} \frac{\partial E_{r}}{\partial r}\right)+\frac{1}{r^{2} \sin ^{2} \theta} \frac{\partial^{2} E_{r}}{\partial \phi^{2}}+k_{2}^{2} E_{r}+k_{3}^{2} E_{r}=0$.

This equation can be satisfied, if

$$
\left\{\begin{array}{c}
\frac{1}{r^{2} \sin ^{2} \theta} \frac{\partial^{2} E_{r}}{\partial \phi^{2}}+k_{2}^{2} E_{r}=0 ; \\
\frac{1}{r^{2}} \frac{\partial}{\partial r}\left(\frac{r^{2} \partial E_{r}}{\partial r}\right)+k_{3}^{2} E_{r}=0
\end{array}\right\}
$$

Thus, initial Helmholtz equation has split into the system of two equations. We substitute in these equations instead of $E_{r}(r, \phi)=f(r) g(\phi)$, (i.e. we are searching the solution as the product of two functions) and divide the first equation by $f(r)$, and the second by $g(\phi)$. We receive

$$
\left\{\begin{array}{l}
\frac{d^{2} g}{d \phi^{2}}+k_{2}^{2} r^{2}\left(\sin ^{2} \theta\right) g=0 ; \\
r^{2} \frac{d^{2} f}{d r^{2}}+2 r \frac{d f}{d r}+k_{3}^{2} r^{2} f=0 .
\end{array}\right\}
$$

Equations (16) and (18) are equivalent to equations (7) и ( $\left.7^{\prime}\right)$, which were received earlier from Maxwell's equations, and

$$
\begin{aligned}
& k_{2}=\omega \sqrt{\varepsilon \mu}=\frac{\omega}{v}=\frac{1}{2 r \sin \theta} ; \\
& k_{1}=k_{2} r \sin \theta=\frac{1}{2}
\end{aligned}
$$

The solution of equation (18) was found earlier, look (13).

$$
g(\phi)=\sin \frac{\phi}{2} .
$$

Let us copy (19) as: $r^{2} \frac{d^{2} f}{d r^{2}}+2 r \frac{d f}{d r}+k_{4}^{2} f=0 ;$

Where

$k_{4}=k_{3} r$

$19^{\prime}$ - centrally symmetric Helmholtz equation. Let us suggest, $k_{4}=k_{3} r$ $k_{3}=\frac{\omega}{v_{r}}$,

Where $\mathrm{v}_{\mathrm{r}}-$ phase velocity of electromagnetic wave in radial direction. As in the central symmetric equation angular dependence is absent, it is logical to assume that

$$
\begin{aligned}
& v_{\mathrm{r}=} v=2 \omega r \sin \theta \\
& \text { at } \theta=\frac{\pi}{2} ; \text { i.e. } \\
& v=2 \omega r \\
& k_{3}=\frac{\omega}{v_{r}}=\frac{1}{2 r} ; \\
& k_{4}=k_{3} r=\frac{1}{2} .
\end{aligned}
$$

Instead of $\left(19^{\prime}\right)$, we are having

$r^{2} \frac{d^{2} f}{d r^{2}}+2 r \frac{d f}{d r}+\frac{1}{4} f=0$.

This is Euler equation, it has the solution

$f=r^{-\frac{1}{2}}\left(C_{1}+C_{2} \ln r\right)$.

Let us converse expression (22).

$f=\frac{\left(C_{1}+C_{2} \ln r\right)}{\sqrt{r}}=\sqrt{\frac{a}{r}}\left(C_{3}+C \ln r\right)=\sqrt{\frac{a}{r}}\left(1+\ln C_{5}+\ln r^{C}\right)=\sqrt{\frac{a}{r}}\left(1+\ln C_{5} r^{C}\right)$.

Here $C_{1}=\sqrt{a} C_{3} ; C_{2}=\sqrt{a} C ; C_{3}=1+\ln C_{5} ; a$-value of radius $r$, at which the rotating monochromatic electromagnetic wave ceases to exist, and $E_{\mathrm{r}}=E_{0} ; f=1$ hence

$$
\begin{aligned}
& 1+\ln C_{5} a^{C}=1 ; \\
& \ln C_{5} a^{C}=0 ; \\
& C_{5} a^{C}=1 ; \\
& C_{5}=\frac{1}{a^{C}} .
\end{aligned}
$$

In view of this,

$$
f=\sqrt{\frac{a}{r}}\left(1+\ln \left(\frac{r}{a}\right)^{C}\right)=\sqrt{\frac{a}{r}}\left(1+C \ln \frac{r}{a}\right) .
$$

Let us designate $C=p$ now

$$
f=\sqrt{\frac{a}{r}}\left(1+p \ln \frac{r}{a}\right) .
$$

Thus, for $E_{\mathrm{r}}$ we are having

$$
E_{r}=E_{0} g(\phi) f(r)=E_{0} \sqrt{\frac{a}{r}}\left(1+p \ln \frac{r}{a}\right) \sin \frac{\phi}{2} .
$$

At $r \rightarrow \infty, E_{r}=0 ; f=0$.

Really

$$
\lim _{r \rightarrow \infty} \frac{\ln r}{\sqrt{r}}=\lim _{r \rightarrow \infty} \frac{1 / r}{1 / 2 \sqrt{r}}=0 .
$$

So that at alteration of $r$ within the interval from 0 to $a, E_{\mathrm{r}}$ would not change its sign, observance of the following requirement is necessary $P \leq 0$ 


$$
\begin{aligned}
& \text { At } r=0, E_{r}=\infty ; f=\infty . \\
& \text { At } E_{r}=E_{0} \sqrt{\frac{a}{r}}\left(1+p \ln \frac{r}{a}\right) \sin \frac{\phi}{2} ;
\end{aligned}
$$

\section{System of Equations for Electron}

Basing on results of the previous section, let us write down expressions for electromagnetic field inside the electron, assuming that it is concentrated inside the orb of radius $a$

$$
\begin{aligned}
& E_{r}=E_{0} \sqrt{\frac{a}{r}}\left(1+p \ln \frac{r}{a}\right) \sin \frac{\phi}{2} ; \\
& H_{\theta}=\frac{E_{0}}{z} \sqrt{\frac{a}{r}}\left(1+p \ln \frac{r}{a}\right) \sin \frac{\phi}{2} .
\end{aligned}
$$

Here $a$ is electron radius, $E_{0}$ amplitude of electric field intensity at $r=a ; \mathrm{z}=$ const characteristic impedance inside the electron, $P$ - unknown coefficient and $P \leq 0$

At that the internal electron medium possesses frequent and spatial dispersion, as well as anisotropy. Dispersion equations have the following appearance [7-11].'

$$
\begin{aligned}
& v_{\mathrm{r}}=v_{\theta}=2 \omega r ; \\
& v_{\phi}=2 r \sin \theta \\
& z_{\mathrm{r}}=z_{\theta}=z_{\phi}=z=\text { const }
\end{aligned}
$$

Here $v_{\mathrm{r}}, v_{\theta}, v_{\phi}$ - phase velocity of rotating monochromatic electromagnetic wave in corresponding direction. In viewed case, the electromagnetic wave is being spread only in the direction $\phi$, and we shall need expressions $v_{\mathrm{r}}$ and $v_{\theta}$ for searching the formulas of dielectric and magnetic permeability, as well as wave numbers of corresponding directions; $z_{\mathrm{r}}, z_{\theta} z_{\phi} z$ characteristic impedances inside the electron; $\varepsilon_{\varphi}, \mathrm{u}$, $\mu_{\varphi}$ were found before, see $\left(12^{\prime}\right)\left(12^{\prime \prime}\right)$

$$
\begin{aligned}
& \varepsilon_{\phi}=\frac{1}{2 \omega r z \sin \theta}=\frac{1}{v_{\phi} z} ; \\
& \mu_{\phi}=\frac{z}{2 \omega r \sin \theta}=\frac{z}{v_{\phi}} .
\end{aligned}
$$

In view of $(24)\left(24^{\prime}\right)\left(24^{\prime \prime}\right)$, let us write down expressions for $\varepsilon_{r^{\prime}}$ $\varepsilon_{\theta}, \mu_{r}, \mu_{\theta}$

$$
\begin{aligned}
& \varepsilon_{r}=\frac{1}{2 \omega r z} ; \\
& \mu_{r}=\frac{z}{2 \omega r} ; \\
& \varepsilon_{\theta}=\frac{1}{2 \omega r z} ; \\
& \mu_{\theta}=\frac{z}{2 \omega r} .
\end{aligned}
$$

From considerations and formulas adduced, it follows that dielectric and magnetic permeability are tensor values.

$$
\|\varepsilon\|=\left|\begin{array}{ccc}
\varepsilon_{r} & 0 & 0 \\
0 & \varepsilon_{\phi} & 0 \\
0 & 0 & \varepsilon_{\theta}
\end{array}\right|=\left|\begin{array}{ccc}
\frac{1}{2 \omega r z} & 0 & 0 \\
0 & \frac{1}{2 \omega r z \sin \theta} & 0 \\
0 & 0 & \frac{1}{2 \omega r z}
\end{array}\right| .
$$

$$
\|\mu\|=\left|\begin{array}{ccc}
\mu_{r} & 0 & 0 \\
0 & \mu_{\phi} & 0 \\
0 & 0 & \mu_{\theta}
\end{array}\right|=\left|\begin{array}{ccc}
\frac{z}{2 \omega r} & 0 & 0 \\
0 & \frac{z}{2 \omega r \sin \theta} & 0 \\
0 & 0 & \frac{z}{2 \omega r}
\end{array}\right| .
$$

Let us find dimensionless wave numbers.

$k_{\phi}=\frac{\omega}{v_{\phi}} r \sin \theta=\frac{\omega r \sin \theta}{2 \omega r \sin \theta}=\frac{1}{2}$;

$k_{\theta}=\frac{\omega}{v_{\theta}} r=\frac{\omega r}{2 \omega r}=\frac{1}{2}$;

$k_{r}=\frac{\omega}{v_{r}} r=\frac{\omega r}{2 \omega r}=\frac{1}{2}$.

Thus

$k_{\phi}=k_{\theta}=k_{r}=\frac{1}{2}$.

Let us remind that in the viewed case, the electromagnetic wave is spread only in the direction of $\phi$

At $r=0$ we are having a special point:

$E_{r}=\infty ; H_{\theta}=\infty ; \quad \rho=\infty ; \quad j_{\phi}=\infty ; \quad\|\varepsilon\|=\infty ; \quad\|\mu\|=\infty$.

Despite of this, all basic electrons' parameters - charge $q$ rest energy $W$, magnetic moment $M$-expressed through integrals by volume from the functions specified above, prove to be finite quantities [12-15]. Look further.

From (5), we find volume charge density inside electron

$$
\begin{aligned}
& \rho=\operatorname{div}\left(\varepsilon_{r} E_{r}\right)=-\frac{\partial}{r}\left[\frac{r E \sin -}{r z}\left(\left(\frac{a}{r}\right)^{\frac{1}{2}}+p\left(\frac{a}{r}\right)^{\frac{1}{2}} \ln \frac{r}{a}\right)\right]= \\
& =\frac{E_{0} \sqrt{a} \sin \frac{\phi}{2}}{2 \omega z r^{2}}\left(\left(\frac{1}{2}+p\right) \frac{1}{\sqrt{r}}+\frac{p}{2 \sqrt{r}} \ln \frac{r}{a}\right) .
\end{aligned}
$$

Integrating $\rho$ on electron's volume, we shall receive this expression for its charge $q$.

$$
\begin{aligned}
& q=\int_{V} \rho d V=\frac{E_{0} \sqrt{a}}{2 \omega z} \int_{0}^{2 \pi} \int_{0}^{\pi} \int_{0}^{a} \frac{\sin \frac{\phi}{2} r^{2} \sin \theta}{r^{2}}\left(\left(\frac{1}{2}+p\right) \frac{1}{\sqrt{r}}+\frac{p}{2 \sqrt{r}} \ln \frac{r}{a}\right) d \phi d \theta d r= \\
& =\frac{4 E_{0} \sqrt{a}}{\omega z}\left(2 \sqrt{a}\left(\frac{1}{2}+p\right)+p \sqrt{a} \ln a-2 p \sqrt{a}-p \sqrt{a} \ln a-p \sqrt{0} \ln 0\right)=\frac{4 E_{0} a}{\omega z} . \text { (26) }
\end{aligned}
$$

On the other hand, from the third integral Maxwell's equation, it is possible to find electron's charge as a stream of vector electric induction $\mathrm{D}$ through the surface of the orb of radius $a$

$$
q=\oint_{S} \varepsilon_{r} E_{r} d S=\int_{0}^{2 \pi} \int_{0}^{\pi} \frac{E_{0} \sin \frac{\phi}{2} a^{2} \sin \theta}{2 \omega z a} d \phi d \theta=\frac{4 E_{0} a}{\omega z} .
$$

As we can see, expressions (26) и (26') are equivalent to each other.

From (1), we obtain expression for current density $j_{\phi}$

$$
j_{\phi}=\frac{1}{r} \frac{\partial}{\partial r}\left(r \frac{E_{0} \sin \frac{\phi}{2}}{z}\left(\sqrt{\frac{a}{r}}+p \sqrt{\frac{a}{r}} \ln \frac{r}{a}\right)\right)=\frac{E_{0} \sqrt{a} \sin \frac{\phi}{2}}{r z}\left(\left(\frac{1}{2}+p\right) \frac{1}{\sqrt{r}}+\frac{p}{2 \sqrt{r}} \ln \frac{r}{a}\right)
$$

From expressions (25), (27) it is visible that in the interval of change of $r$ from 0 to $a, \rho$ and $j_{\phi}$ once change the sign. It can be explained by the fact that in the viewed structure, the substantial role is played by the 
rotating monochromatic electromagnetic wave, and the space charge density and electric current density - are auxiliary or even fictitious quantities in the sense that inside the particle there is neither any charged substance nor its motion [16]. Inside the electron, it is not the charge that is the source of electric field, but electric field is the source of the charge. In its turn, it is not the electric current that is the source of magnetic field, but magnetic field is the source of the electric current [17-22]. Thus, a deduction about vector nature of elementary charge can be made.

Now we shall determine electron's rest energy as electromagnetic wave energy inside a particle.

$$
W=\int_{V} w d V
$$

Here $w$ - is volume density of electromagnetic wave energy,

$w=\frac{\Pi}{v}$, where

$\Pi$ - Póinting vector,

$\Pi=\left[E_{r} H_{\theta}\right]$,

$v_{\phi}$-phase velncity of electromagnetic wave in direction of $v \phi$.

$v_{\phi}=2 \omega r \sin \theta$

$W=\int_{0}^{2 \pi} \int_{0}^{\pi} \int_{0}^{a} \frac{E_{0}^{2} \sin ^{2} \frac{\phi}{2}}{2 \omega r z \sin \theta}\left(\frac{a}{r}+2 p \frac{a}{r} \ln \frac{r}{a}+p^{2} \frac{a}{r} \ln ^{2} \frac{r}{a}\right) r^{2} \sin \theta d \phi d \theta d r=$

$=\frac{\pi^{2} E_{0}{ }^{2} a}{2 \omega z}\left(a+2 p a \ln a-2 p 0 \ln 0-2 p a-2 p a \ln a+p^{2} a\left(2-0 \ln ^{2} 0+2 * 0 \ln 0\right)\right)=$

$=\frac{\pi^{2} E_{0}^{2} a^{2}}{2 \omega z}\left(1-2 p+2 p^{2}\right)$.

$\frac{\pi^{2} E_{0}^{2} a^{2}}{2 \omega z}\left(1-2 p+2 p^{2}\right)=\hbar \omega$

Here $\hbar$ is Planck's constant.

We shall be searching electron's magnetic moment in the form of a sum. $M=M_{m}+M_{L}$

$$
M=M_{m}+M_{L}
$$

Where $M_{m}$ - is magnetic moment, created by volumetric current; $M_{L}$-magnetic moment, attributed to impulse moment, i.e. to rotation.

$$
M_{L}=\gamma L
$$

Where $\gamma$-gyromagnetic ratio; $L$-impulse moment of electron.

Basing on Barnett effect, we are making a supposition, that the impulse moment, attributed to rotation, creates additional magnetic moment $[21,23]$.

Being aware of the fact that electron's impulse moment is equal $\frac{\hbar}{2}$, from $\left(28^{\prime}\right)$ we find expression for $\mathrm{L}$.

$$
\begin{aligned}
& L=\frac{\pi^{2} E_{0}^{2} a^{2}}{4 \omega^{2} z}\left(2 p^{2}-2 p+1\right) ; \\
& M_{L}=\frac{\gamma \pi^{2} E_{0}^{2} a^{2}\left(2 p^{2}-2 p+1\right)}{4 \omega^{2} z} ; \\
& \text { or } M_{L}=\gamma \frac{\hbar}{2} .
\end{aligned}
$$

Let us calculate $M_{m}$ as electric current magnetic moment in volume $\mathrm{V}$, relating to axis $\mathrm{z}$ by the formula:

$$
M_{\grave{o}}=\frac{1}{2} \int_{V}\left[r_{z}, j_{\phi}\right] d V .
$$

See for instance [3], page 111, where $r_{\mathrm{z}}$ - distance to axis $\mathrm{z}$,

$r_{z=} r \sin \theta$

$$
\begin{aligned}
& M_{\dot{o}}=\frac{1}{2} \int_{0}^{2 \pi} \int_{0}^{\pi} \int_{0}^{a} \frac{r \sin \theta E_{0} \sqrt{a} \sin \frac{\phi}{2} r^{2} \sin \theta}{z r^{\frac{3}{2}}} \\
& \left(p+\frac{1}{2}+\frac{p^{\prime}}{2} \ln \frac{r}{a}\right) d \phi d \theta d r=\frac{\pi E_{0} a^{3}}{z}\left(\frac{8 p+5}{25}\right) . \\
& M=M_{\dot{o}}+M_{L}=\frac{\pi E_{0} a^{3}}{z}\left(\frac{8 p+5}{25}\right)+\gamma \frac{\hbar}{2} .
\end{aligned}
$$

Or

$M=\frac{\pi E_{0} a^{2}}{z}\left[a\left(\frac{8 p+5}{25}\right)+\frac{\gamma \pi E_{0}}{4 \omega^{2}}\left(1-2 p+2 p^{2}\right)\right]$. electron.

Thus, we have received the system of algebraic equations for

$$
\left\{\begin{array}{l}
\frac{4 E_{0} a}{\omega z}=-e ; \\
\frac{\pi E_{0} a^{3}}{z}\left(\frac{8 p+5}{25}\right)+\frac{\gamma \hbar}{2}=-1,0011595 \frac{e \hbar}{2 m} \\
\frac{\pi^{2} E_{0}^{2} a^{2}}{2 \omega z}\left(1-2 p+2 p^{2}\right)=\hbar \omega ;
\end{array}\right.
$$

Here $e$ - charge of electron, $m$ - it's mass.

Three equations contain five unknown quantities: $E_{0}, a, z, p, \gamma$ Let us add this system with equations, which we shall receive from boundary conditions.

$$
\begin{aligned}
& \text { At } r=a, R=a \\
& \varepsilon_{r} E_{0}=\varepsilon_{0} E_{\text {внеин. }}
\end{aligned}
$$

In the exterior area, the same as and in the interior area, electric field intensity possesses only radial component. Here R - distance from electron's center to the observation point in the exterior area, $\varepsilon_{0}$ vacuum dielectric permeability [24].

Further. $H_{0}=\frac{E_{0}}{z}=H_{\text {внеин. }}$

In the exterior area, the same as and in the interior area, magnetic field intensity possesses only meridional component.

It is obvious that

$\varepsilon_{r} \geq \varepsilon_{0}$,

then from (33) follows:

$E_{0} \leq E_{\text {внеин }}$

On the other hand it is known that the electric field, having passed through dielectric layer, cannot increase, therefore

$$
E_{0} \geq E_{\text {внеин. }}
$$

In other words, correlations $\left(33^{\prime}\right)\left(33^{\prime \prime}\right)\left(33^{\prime \prime \prime}\right)$ will be simultaneously executed only in one case, if

$$
\varepsilon_{\mathrm{r}=} \varepsilon_{0}
$$

$$
E_{\text {внеин. }}=E_{0} \text {. }
$$

Now under Biot-Savart's law, we are finding magnetic field in the exterior area. 
$B_{\text {внеин. }}=\frac{1}{4 \pi} \int_{V} \frac{\left[j_{\phi} R\right] \mu_{\phi}}{R^{3}} d V$.

In last expression we substitute $\left(12^{\prime \prime}\right)$ and (27).

$B_{\text {вней. }}=\frac{E_{0} \sqrt{a}}{4 \pi R^{2} z} \int_{0}^{2 \pi} \int_{0}^{\pi a} \int_{0}^{a} \frac{z \sin \frac{\phi}{2}\left[\left(\frac{1}{2}+p\right) \frac{1}{\sqrt{r}}+\frac{p}{2 \sqrt{r}} \ln \frac{r}{a}\right]}{2 \omega r^{2} \sin \theta} r^{2} \sin \theta d \phi d \theta d r=$

$=\frac{E_{0} \sqrt{a}}{2 \omega R^{2}}\left[\left(\frac{1}{2}+p\right) 2 \sqrt{a}-p \sqrt{a} \ln a+p \sqrt{a} \ln a-p \sqrt{0} \ln 0-2 p \sqrt{a}\right]=\frac{E_{0} a}{2 \omega R^{2}}$.

$H_{\text {внеин. }}=\frac{B_{\text {внеин. }}}{\mu_{0}}=\frac{E_{0} a}{2 \mu_{0} \omega R^{2}}$.

At $r=a R=a$

$H_{\text {внутр. }}=\frac{E_{0}}{z}=H_{\text {внеин. }}$

$\frac{E_{0}}{z}=\frac{E_{0} a}{2 \mu_{0} \omega a^{2}}=\frac{E_{0}}{2 \mu_{0} \omega a} ;$

$z=2 \mu_{0} \omega a$.

On the other hand, from $\left(24^{\prime \prime \prime}\right)$

$z=2 \mu_{\theta} \omega r$.

At $r=a$

$z=2 \mu_{\theta} \omega a .\left(39^{\prime}\right)$

We substitute in (39).

$2 \mu_{\theta} \omega a=2 \mu_{0} \omega a$;

$\mu_{\theta}=\mu_{0}$

Thus, at $r=a$

$\varepsilon_{r}=\varepsilon_{\theta}=\varepsilon_{0} ;$

$\mu_{\theta}=\mu_{r}=\mu_{0}$

$v_{r}=v_{\theta}=2 \omega a=\frac{1}{\sqrt{\mu_{0} \varepsilon_{0}}}=c$.

Here $c$ - velocity of light, $\omega=7,7634421^{\star} 10^{20} \mathrm{~Hz}$ - Compton circular frequency of electron.

$a=\frac{c}{2 \omega}=0,1930796 * 10^{-12}(\mathrm{~m})$.

As it is known, atom's radius approximately equals to $10^{-10} \mathrm{~m}$, volume of atom $-4,18879^{\star} 10^{-30} \mathrm{~m}^{3}$. We found, that radius of electron equals to $1,930796^{\star} 10^{-13} \mathrm{~m}$, volume of electron $-3,0150724^{\star} 10^{-38} \mathrm{~m}^{3}$. That is one electron occupies $0,7197955^{\star} 10^{-8}$ from atom's volume and, for example, 100 electrons (as in atoms located at the end of the periodic system) occupy $0,7197955^{\star} 10^{-6}$ from atom's volume $[8,25]$.

We substitute (42) в (39).

$z=\frac{2 \mu_{0} \omega c}{2 \omega}=\sqrt{\frac{\mu_{0}}{\varepsilon_{0}}}=376,73032(\mathrm{Ohm})$.

Let us solve the system (30), (31), (32), taking into account (42) and (43)

$$
\begin{aligned}
& \frac{4 E_{0} c}{\omega \sqrt{\frac{\mu_{0}}{\varepsilon_{0}}} 2 \omega}=-e ; \\
& E_{0}=-\frac{\omega^{2} \mu_{0} e}{2} .
\end{aligned}
$$

$$
\begin{aligned}
& \frac{\pi E_{0}}{8 \omega^{3} \mu_{0}^{2} \varepsilon_{0}}\left(\frac{8 p+5}{25}\right)+\frac{\gamma \pi^{2} E_{0}^{2}\left(1-2 p+2 p^{2}\right)}{16 \omega^{4} \mu_{0}^{3 / 2} \varepsilon_{0}^{1 / 2}}=-1,0011595 \frac{e \hbar}{2 m} . \\
& \frac{\pi^{2} E_{0}{ }^{2}}{8 \omega^{3} \mu_{0}^{3 / 2} \varepsilon_{0}^{1 / 2}}\left(1-2 p+2 p^{2}\right)=\hbar \omega .
\end{aligned}
$$

We substitute $\left(30^{\prime}\right)$ in $\left(32^{\prime}\right)$.

$p^{2}-p+\frac{1}{2}-\frac{16 \hbar \varepsilon_{0}^{1 / 2}}{\pi^{2} e^{2} \mu_{0}^{1 / 2}}=0 ;$

$p_{1}=4,6747427$

$p_{2}=-3,6747427$.

$P$ must be negative, therefore we select

$p_{2}=p=-3,6747427$.

We substitute $\left(30^{\prime}\right)$ in $\left(31^{\prime}\right)$

$-\frac{\pi e}{16 \omega \mu_{0} \varepsilon_{0}}\left(\frac{8 p+5}{25}\right)+\frac{\pi^{2} \gamma e^{2} \mu_{0}^{1 / 2}}{64 \varepsilon_{0}^{1 / 2}}\left(1-2 p+2 p^{2}\right)=-1,0011595 \frac{e \hbar}{2 m}$.

We substitute $p$ meaning in (31") and find $\gamma$

$\gamma=-0,2434911 * 10^{12}\left(\frac{1}{T^{*} S}\right)$.

From solution of equation (31), it is visible that two components of magnetic moment of electron $M_{m} и M_{L}$ are directed to opposite sides and $M_{L} \succ M_{m}$

Let us also calculate numerical value of $E_{0}$ by formula ( $\left.30^{\prime}\right)$

$$
E_{0}=-6,0673455 * 10^{16}\left(\frac{\mathrm{V}}{\mathrm{m}}\right) \text {. }
$$

"Dimensions" of electron for the present are not discovered by experimental way, though precision of measuring is led to $10^{-18} \mathrm{~m}$. Within the framework of the model considered it may be explained by the next way: electron is not hard particle with this quantity of vector E, which exist inside it, unlike from proton and neutron, quantity of vector $\mathrm{E}$ inside which approximately $10^{7}$ times as much [26-31].

For positron, the system of equations will take a somewhat different view.

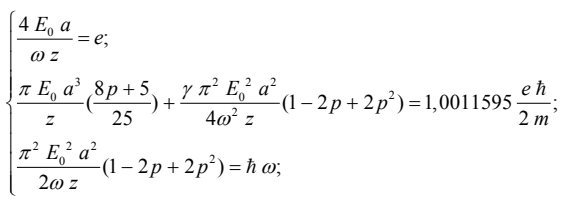

Boundary conditions are the same as for electron. Hence

$$
\begin{aligned}
& z=\sqrt{\frac{\mu_{0}}{\varepsilon_{0}}} ; \\
& a=\frac{c}{2 \omega}=0,1930796 * 10^{-12}(\mathrm{~m}) .
\end{aligned}
$$

The system of equations (44), (45), (46) with exactness to a sign, has the same solutions, as the system (30), (31), (32).

$$
\begin{aligned}
& E_{0}^{e+}=-E_{0}^{e}=6,0673455 * 10^{16}\left(\frac{V}{m}\right) ; \\
& \gamma_{e+}=-\gamma_{e}=0,2434911 * 10^{12}\left(\frac{1}{T^{*} s}\right) ; \\
& p_{e+}=-p_{e}=-3,6747427 .
\end{aligned}
$$

\section{System of Equations for Proton}

By applying reasoning and mathematical calculations of the 
previous section in relation to proton, we shall receive the relevant system of equations.

$$
\left\{\begin{array}{l}
\frac{4 E_{0} a}{\omega z}=e \\
\frac{\pi E_{0} a^{3}}{z}\left(\frac{8 p+5}{25}\right)+\frac{\gamma \pi^{2} E_{0}^{2} a^{2}\left(1-2 p+2 p^{2}\right)}{4 \omega^{2} z}=-2,7928475 \frac{e \hbar}{2 m} \\
\frac{\pi^{2} E_{0}^{2} a^{2}}{2 \omega z}\left(1-2 p+2 p^{2}\right)=\hbar \omega
\end{array}\right\}
$$

Here corresponding letters mean parameters of proton.

Boundary conditions: at $r=a$

$\varepsilon_{r}=\varepsilon_{\theta}=\varepsilon_{0}$

$\mu_{\theta}=\mu_{r}=\mu_{0}$

Hence

$z=\sqrt{\frac{\mu_{0}}{\varepsilon_{0}}}$

$a=\frac{c}{2 \omega}=1,0515447 * 10^{-16} \mathrm{~m}$

Here: $\omega=1,425486^{\star} 10^{24} \mathrm{~Hz}$ - Compton circular frequency of proton $[32,33]$.

Solving the system (47), (48), (49), we shall receive

$$
\begin{aligned}
& E_{0}=2,0455794 * 10^{23}\left(\frac{V}{m}\right) ; \\
& p=-3,6747427 ; \\
& \gamma=-2,3081218 * 10^{8}\left(\frac{1}{T * s}\right) .
\end{aligned}
$$

From the solution of equation (48) it is visible that two components of proton's magnetic moment $M_{m}$ и $M_{L}$ have identical direction, and $M_{L} \succ M_{m}$

Let us write down the system of equations for antiproton explained in [34].

$$
\begin{aligned}
& \frac{4 E_{0} a}{\omega z}=-e ; \\
& \frac{\pi E_{0} a^{3}}{z}\left(\frac{8 p+5}{25}\right)+\frac{\gamma \pi^{2} E_{0}^{2} a^{2}\left(1-2 p+2 p^{2}\right)}{4 \omega^{2} z}=2,7928475 \frac{e \hbar}{2 m} ; \\
& \frac{\pi^{2} E_{0}^{2} a^{2}}{2 \omega z}\left(1-2 p+2 p^{2}\right)=\hbar \omega .
\end{aligned}
$$

Boundary conditions: at $r=a$

$\varepsilon_{r}=\varepsilon_{\theta}=\varepsilon_{0}$;

$\mu_{r}=\mu_{\theta}=\mu_{0}$

Hence

$$
\begin{aligned}
& z=\sqrt{\frac{\mu_{0}}{\varepsilon_{0}}} ; \\
& a=\frac{c}{2 \omega}=1,0515447 * 10^{-16}(\mathrm{~m}) .
\end{aligned}
$$

System of equations (50), (51), (52) with exactness to a sign has the same solutions, as system (47), (48), (49).

$$
\begin{aligned}
& E_{0}^{\tilde{p}}=-E_{0}^{p}=-2,0455794 * 10^{23}\left(\frac{V}{m}\right) ; \\
& \gamma_{\tilde{p}}=-\gamma_{p}=2,3081218 * 10^{8}\left(\frac{1}{T^{*} s}\right) ; \\
& p_{\tilde{p}}=p_{p}=p_{e}=-3,6747427 .
\end{aligned}
$$

\section{System of equations for neutron}

$$
\begin{aligned}
& E_{r}=E_{0} \sqrt{\frac{a}{r}}\left(1+p \ln \frac{r}{a}\right) \sin \phi ; \\
& H_{\theta}=\frac{E_{0}}{z} \sqrt{\frac{a}{r}}\left(1+p \ln \frac{r}{a}\right) \sin \phi .
\end{aligned}
$$

Along each parallel, exactly one wave length is kept within. In this case: $v_{\phi}=\omega r \sin \theta$;

$\varepsilon_{\phi}=\frac{1}{\omega r z \sin \theta}$;

$\mu_{\phi}=\frac{z}{\omega r \sin \theta}$.

$v_{r}=2 \omega r$

$\varepsilon_{r}=\frac{1}{2 \omega r z}$;

$\mu_{r}=\frac{z}{2 \omega r}$.

$v_{\theta}=v_{r}=2 \omega r$

$\varepsilon_{\theta}=\varepsilon_{r}=\frac{1}{2 \omega r z} ;$

$$
\mu_{\theta}=\mu_{r}=\frac{z}{2 \omega r}
$$

In other words, anisotropy is taking place, $\varepsilon$ and $\mu$ are tensor quantities.

$$
\begin{aligned}
& \|\varepsilon\|=\left|\begin{array}{ccc}
\varepsilon_{r} & 0 & 0 \\
0 & \varepsilon_{\phi} & 0 \\
0 & 0 & \varepsilon_{\theta}
\end{array}\right|=\left|\begin{array}{ccc}
\frac{1}{2 \omega r z} & 0 & 0 \\
0 & \frac{1}{\omega r z \sin \theta} & 0 \\
0 & 0 & \frac{1}{2 \omega r z}
\end{array}\right| . \\
& \|\mu\|=\left|\begin{array}{ccc}
\mu_{r} & 0 & 0 \\
0 & \mu_{\phi} & 0 \\
0 & 0 & \mu_{\theta}
\end{array}\right|=\left|\begin{array}{ccc}
\frac{z}{2 \omega r} & 0 & 0 \\
0 & \frac{z}{\omega r \sin \theta} & 0 \\
0 & 0 & \frac{z}{2 \omega r}
\end{array}\right| .
\end{aligned}
$$

Here and further, corresponding letters mean parameters of neutron.

Let us find rest energy of neutron.

$$
\begin{aligned}
& W=\int_{V} w d V=\int_{V} \frac{\left[E_{r} H_{\theta}\right]}{v_{\phi}} d V= \\
& =\int_{0}^{2 \pi} \int_{0}^{\pi} \int_{0}^{a} \frac{E_{0}{ }^{2} \frac{a}{r} \sin ^{2} \phi}{\omega r z \sin \theta}\left(1+2 p \ln \frac{r}{a}+p^{2} \ln ^{2} \frac{r}{a}\right) r^{2} \sin \theta d \phi d \theta d r= \\
& =\frac{\pi^{2} E_{0}^{2} a}{\omega z}\left[a+2 p a \ln a-2 p 0 \ln 0-2 p a-2 p a \ln a+p^{2} a\left(\ln ^{2} 1-2 \ln 1+2\right)-p^{2} a\left(0 \ln ^{2} 0-2 * 0 \ln 0\right)\right]= \\
& =\frac{\pi^{2} E_{0}^{2} a^{2}\left(1-2 p+2 p^{2}\right)}{\omega z} .
\end{aligned}
$$

Further. Charge of neutron is equal to zero.

$q=\oint_{S} \varepsilon_{r} E_{r} d S=0$. 
Really,

$\int_{0}^{2 \pi} \int_{0}^{\pi} \frac{E_{0} \sin \phi}{2 \omega a z} a^{2} \sin \theta d \phi d \theta=0$

It is obvious that

$\int_{0}^{\pi} \int_{0}^{\pi} \frac{E_{0} a \sin \phi \sin \theta}{2 \omega z} d \phi d \theta=-\int_{\pi}^{2 \pi} \int_{0}^{\pi} \frac{E_{0} a \sin \phi \sin \theta}{2 \omega z} d \phi d \theta \neq 0$.

It is logical to assume that

$\int_{0}^{\pi} \int_{0}^{\pi} \frac{E_{0} a \sin \phi \sin \theta}{2 \omega z} d \phi d \theta=-\int_{\pi}^{2 \pi} \int_{0}^{\pi} \frac{E_{0} a \sin \phi \sin \theta}{2 \omega z} d \phi d \theta=e$

Then

$\frac{2 E_{0} a}{\omega z}=e$.

Magnetic moment for neutron will be searched as the sum:

$M=M_{m}+M_{L}$

Where $M_{m}$ - magnetic moment created by volume current; $M_{L}$ magnetic moment, attributed to impulse moment, i.e. to rotation.

$M_{m}=\frac{1}{2} \int_{0}^{2 \pi} \int_{0}^{\pi} \int_{0}^{a} \frac{E_{0} \sqrt{a} r \sin \theta \sin \phi}{z r^{3 / 2}}\left(p+\frac{1}{2}+\frac{1}{2} p \ln \frac{r}{a}\right) r^{2} \sin \theta d \phi d \theta d r=0 ;$

as

$\int_{0}^{2 \pi} \sin \phi d \phi=0$

$M_{L}=\gamma L$

$M_{L}=\gamma \frac{\hbar}{2}=M$.

Now we shall write down the system of equations for neutron.

$\left\{\begin{array}{l}\frac{2 E_{0} a}{\omega z}=e \\ \frac{\pi^{2} E_{0}^{2} a^{2}}{\omega z}\left(1-2 p+2 p^{2}\right)=\hbar \omega \\ \gamma \frac{\hbar}{2}=-0,96623707 * 10^{-26} .\end{array}\right.$

Boundary conditions: at $r=a$

$\varepsilon_{r}=\varepsilon_{\theta}=\varepsilon_{0}$;

$\mu_{r}=\mu_{\theta}=\mu_{0}$;

Hence

$z=\sqrt{\frac{\mu_{0}}{\varepsilon_{0}}}$.

From (54) и (54') follows that

$\varepsilon_{\phi}=\varepsilon_{r} \frac{2}{\sin \theta}$

and from (54) и (54')that

$\mu_{\phi}=\mu_{\theta} \frac{2}{\sin \theta}$.

$v_{\phi}=\omega a \sin \theta=\frac{1}{\sqrt{\varepsilon_{\phi} \mu_{\phi}}}=\frac{1}{\sqrt{\varepsilon_{0} \mu_{0} \frac{4}{\sin ^{2} \theta}}}=\frac{c \sin \theta}{2}$.

So

$$
\begin{aligned}
& \omega a=\frac{c}{2} ; \\
& a=\frac{c}{2 \omega}=1,0500973 * 10^{-16}(\mathrm{~m}) .
\end{aligned}
$$

Here $\omega=1,4274508^{\star} 10^{24} \mathrm{~Hz}$ - Compton circular frequency of neutron.

Let us solve system $(56)\left(55^{\prime}\right)\left(57^{\prime}\right)$

$E_{0}=\frac{e \omega^{2} \sqrt{\frac{\mu_{0}}{\varepsilon_{0}}}}{c}$

$E_{0}=E_{0}^{n}=4,1024444 * 10^{23}\left(\frac{V}{m}\right)$.

We substitute $\left(56^{\prime \prime}\right) \mathrm{B}\left(55^{\prime}\right)$

$$
\begin{aligned}
& p^{2}-p+0,5-\frac{2 \hbar}{\pi^{2} e^{2} \sqrt{\mu_{0} / \varepsilon_{0}}}=0 ; \\
& p_{1}=1,8999321 ; \\
& p_{2}=-0,8999321 ;
\end{aligned}
$$

$P$ must be negative, therefore we select

$p_{2}=p_{n}=-0,8999321$.

From (57') we find $\gamma$

$\gamma=-1,8324711 * 10^{8}\left(\frac{1}{T^{*} S}\right)$.

Let us write down the system of equations for antineutron.

$$
\left\{\begin{array}{l}
\frac{2 E_{0} a}{\omega z}=e ; \\
\frac{\pi^{2} E_{0}^{2} a^{2}}{\omega z}\left(1-2 p+2 p^{2}\right)=\hbar \omega ; \\
\gamma \frac{\hbar}{2}=0,96623707 * 10^{-26} .
\end{array}\right\}
$$

Boundary conditions are the same, as at neutron, hence

$$
\begin{aligned}
& z=\sqrt{\frac{\mu_{0}}{\varepsilon_{0}}} ; \\
& a=\frac{c}{2 \omega}=1,0500973 * 10^{-16}(\mathrm{~m}) .
\end{aligned}
$$

The last system with exactness to a sign has the same solutions, as system $(56)\left(55^{\prime}\right)(57)$

$$
\begin{aligned}
& E_{0}^{\tilde{n}}=E_{0}^{n}=4,1024444 * 10^{23}\left(\frac{V}{m}\right) ; \\
& p_{\tilde{n}}=p_{n}=-0,8999321 ; \\
& \gamma_{\tilde{n}}=-\gamma_{n}=1,8324711 * 10^{8}\left(\frac{1}{T * s}\right) .
\end{aligned}
$$

\section{Conclusion}

Within the framework of the model, which is considered, electron, proton and neutron represent a monochromatic electromagnetic wave of corresponding frequency spread along parallels inside the spherical area, i.e. a wave, rotating around some axis. At that along each parallel, exactly half of wave length for electron and proton and exactly one wave length for neutron, is kept within, thus this is rotating soliton. This is caused by presence of spatial dispersion and anisotropy of a 
strictly defined type inside the particles. In electron vector $\mathrm{E}$ is directed to center of particle, that correspond to negative charge, and in proton vector $\mathrm{E}$ is directed from center of particle, that correspond to positive charge. Thus, by natural way, all basic parameters of particles are obtained: charge, rest energy, mass, radius, magnetic moment and spin, that is confirmed by mathematical expressions, which are discovered.

\section{References}

1. Sarycheva LI (2000) Structure of matter. Soros educational, Russia.

2. DI Blokhintsev (1983) Principles of quantum mechanics.

3. Born M (1967) Atomic physics.

4. Bredov MM, Rumyantsev VV, Toptygin IN (1985). possibility of measuring the thermal vibration spectrum g (\$ omega \$) using coherent inelastic neutron scattering from a polycrystalline sample. Inst of Semiconductors, Leningrad.

5. Vinogradova MB, Rudenko OV, Sukhorukov AP (1990) Theory of waves.

6. Vlasov AD, PMurin B (1990) Physical quantities units in science and technology.

7. Vladimirov VS, Jeffrey A, Schroeck FE (1971) Equations of mathematical physics. American Journal of Physics 39: 1548-1548.

8. Kalashnikov SG (1985) Electricity.

9. Kamke E (1976) Reference manual on ordinary differential equations. Campman and Hall/CRC

10. Landau LD, Lifshits EM (1989) Quantum mechanics. Non-relativistic theory Physics Today 11: $56-60$

11. Landau LD, Lifshits EM (1973) Field theory.

12. Landau LD, Lifshits EM (1982) Electrodynamics of continuous mediums

13. Prudnikov AP, Yu A, Brychkov O, Marychev I (1981) Integrals and series. Elementary functions. Gordon and Breach Science Publishers, UK.

14. Taylor B, Parker V, Langerberg D (1991) Fundamental constants and quantum electrodynamics. Doklady Akademii Nauk Ukrain 5: 40-45.

15. Terletskiy YP, Yu P Rybakov (1990) Electrodynamics.

16. Turanyanin D (2004) On interaction of motional masses. Journal of Theoretics.

17. Xu Z, Buehler MJ (2010) Interface structure and mechanics between graphene and metal substrates: a first-principles study. Journal of Physics: Condensed Matter 22: 45-56.

18. Kyriakos AG (2003) The electrodynamics form concurrent to the Dirac electron theory. Physics Essays 16: 365-374.

19. Kyriakos AG (2005) The massive neutrino-like particle of the non-linear electromagnetic field theory.

20. Kyriakos AG (2004) Yang-Mills equation as the equation of the superposition of the non-linear electromagnetic waves.

21. Kyriakos AG (2004) Non-linear Theory of quantized Electromagnetic Field equivalent to the Quantum Field Theory.

22. Ivanov IP (2003) Quark model is not quite correctly. Scientific Russia.

23. Kopelyowicz $\mathrm{V}$ Topologic soliton models of baryons and its predictions. Scientific Russia.

24. Diakonov D, Petrov V, Polyakov M Unpolarized and polarized quark distributions in the large- $\mathrm{N}_{c}$ limit $\mathrm{Z}$. Phys Rev D.

25. Skyrme THR (1962) A Non-Linear Field Theory. Nucl Phys 31: 556.

26. Polyakov M (2000) Infinite conformal symmetry in two-dimensional quantum field theory. Eur Phys J 241: 333-380.

27. Nakano $T$ (2003) Evidence for Narrow $S=+1$. Baryon Resonance in Photoproduction from Neutron. Phys Rev Lett.

28. Polyakov MV, Rathke A (2003) on photoexcitation of baryon antidecuplet. Eur Phys J A 18: 691-695.

29. Diakonov D, Petrov V (1985) Baryons as solitons. Elementary particles. Moscow Energoatomisdat.

30. Chemtob M (1985) Skyrme model of baryon octet and decuplet. Nucl Phys B 256: 600-608.

31. Yi SD, Onoda S, Nagaosa N, Han JH (2009) Skyrmions and Anomalous Hall Effect in a Dzyloshinskii-Moriya Spiral Magnet. Physical Review B.

32. Walliser H, Eckart G (1992) baryon resonance as fluctuations of the skyrme soliton. Nuclear physics a 429: 514-526.

33. Diakonov D, Petrov V, Polyakov M (1997) Exotic Anti-Decuplet of Baryons: Prediction from Chiral Solitons. Z Phys A 359: 305-314.

34. Ivanov IP (2006) Last days of Standard Model?
Citation: Sladkov P (2016) Solitonic Model of the Electron, Proton and Neutron J Phys Math 7: 193. doi: 10.4172/2090-0902.1000193
OMICS International: Open Access Publication Benefits \&

\section{Features}

Unique features:

- Increased global visibility of articles through worldwide distribution and indexing

- Showcasing recent research output in a timely and updated manner

Special issues on the current trends of scientific research

Special features:

- 700+ Open Access Journals

$50,000+$ editorial team

Rapid review process

Quality and quick editorial, review and publication processing

Indexing at major indexing services

Sharing Option: Social Networking Enabled

Authors, Reviewers and Editors rewarded with online Scientific Credits

Better discount for your subsequent articles

Submit your manuscript at: http://omicsonline.com/open-access/physical-mathematics.php 